\title{
Des entreprises qui affrontent la compétition en territoire rural acadien
}

\author{
Éric Forgues*, Omer Chouinard** et Danièle Courchesne** \\ *Institut canadien de recherche sur les minorités linguistiques \\ ** Université de Moncton
}

\section{Contexte : des entreprises qui affrontent la compétition en territoire rural acadien}

Le présent article fait suite à une étude que nous avons réalisée en 2006-2007 sur l'association de plusieurs boucanières dans la région rurale de Cap-Pelé à $60 \mathrm{~km}$ de Moncton au Nouveau-Brunswick ${ }^{1}$. Les observateurs de cette industrie locale savaient qu'elles éprouvaient plusieurs difficultés en raison de la forte compétition qui s'exerçait entre elles. L'idée de former une association n'est pas nouvelle. Plusieurs tentatives ont été faites vainement dans le passé. En 2003, le propriétaire d'une boucanière a eu l'idée d'aller chercher quelqu'un à l'extérieur de l'industrie du hareng fumé ${ }^{2}$ qui, de son propre aveu, ne connaissait rien à ce domaine et ne voyait pas comment il pouvait apporter quelque chose de neuf ${ }^{3}$. Pourtant, non

\section{La coopération interentreprises (CIE)}

La création de l'entreprise Les exportations de Harengs Cap-Pelé Inc. représente une forme de coopération entre des entreprises qui visent à mettre un terme à la guerre des prix que se livraient les boucanières et à stabiliser ainsi le marché en créant une entente qui impose des quotas de production pour chaque boucanière. Les boucanières signataires de l'entente de coopération vendent leur produit à cette entreprise, laquelle s'occupe de la mise en marché. La première année, les quotas étaient de 50000 boîtes de harengs, alors que, pour plusieurs, leur capacité pouvait être le seulement a-t-il réussi à créer une association regroupant 18 boucanières sur 25, Les exportations de Harengs Cap-Pelé Inc, mais plus de trois années plus tard, l'association existe toujours. Nous avons voulu comprendre comment on est passé d'une situation de forte concurrence, qui menait littéralement plusieurs boucanières à la faillite, à une situation de coopération entre elles. Quels éléments permettent de comprendre cette transition sociale ? Ce type de question est au cœur de notre démarche de recherche qui inscrit d'emblée l'économie dans la société, mais qui y perçoit également des éléments de production du social (Lévesque et al, 2001) ${ }^{4}$.

double 5 . Par la suite, les quotas ont mieux reflété la capacité de production des entreprises. Chaque boucanière est assurée de voir son produit se vendre à un prix identique pour tous. Ce système permet de réduire la pression exercée sur les boucanières qui cherchaient à devancer la production des autres boucanières afin d'obtenir de meilleurs prix. En outre, il réduit l'incertitude entourant les prix de vente, car même si une boucanière s'entend sur un prix avec un acheteur, celui-ci peut revenir pour lui dire qu'il obtient un meilleur prix avec une autre boucanière, 
l'incitant ainsi à réduire son prix. Les premiers vendeurs avaient un meilleur prix, mais les autres exerçaient une pression à la baisse sur les prix. L'association d'entreprises assure chaque membre qu'il pourra écouler sa production.

«Des entreprises concurrentes dans un même secteur peuvent être conduites à envisager des stratégies collectives et à gérer ainsi leurs interdépendances (...) en unissant leur destin » (Yami, 2003 : 166). La littérature montre que plusieurs facteurs peuvent inciter les entreprises à s'associer. Pour Niosi (2001), l'objectif consiste à réduire l'incertitude. L'incertitude est généralement associée à l'impossibilité de prévoir les comportements d'autres acteurs dans un contexte où ces comportements influent sur l'environnement des entreprises. Cette incertitude s'accroît lorsque chaque acteur poursuit un intérêt qui est le sien sans s'allier aux autres acteurs, les plaçant dans des situations de compétition et de concurrence. Elle peut être réduite lorsque les acteurs coordonnent leurs activités. L'intérêt que présente la CIE pour les acteurs est qu'elle permet de mieux atteindre leur objectif. Dans notre cas, la réduction de l'incertitude visait à mieux planifier la production et stabiliser les prix et les ventes.

Niosi (2001) précise que, dans les ententes de CIE,

1) les entreprises conservent leur autonomie,

2) le partage des capitaux engage les ressources des partenaires et

3) l'objectif à atteindre demande du temps, de l'innovation et un développement.

Les accords passés entre les entreprises se concrétisent dans la forme d'un contrat qui scelle l'alliance entre les entreprises et définit à la fois sa portée, les conditions et les règles à respecter de même que ses limites. Selon Avadikyan et al. (2001).

Le contrat permet d'orienter les comportements des acteurs de façon à éviter qu'ils se nuisent les uns les autres. Toutefois, il ne suffirait pas seul à garantir ce résultat. Certains auteurs estiment que la confiance mutuelle provient, entre autres, de l'enchâssement social de leurs activités économiques. En fait, plus la confiance est réciproque, moins le contrat formel doit être précis et moins il intervient dans la réduction de l'incertitude et des comportements opportunistes individuels. (Blumberg, 2001).

Les liens sociaux, les règles informelles de réciprocité, la confiance, la réputation, la parole donnée et le serrement de mains forment un capital social qui permet aux acteurs de coordonner leurs activités. L'enchâssement des activités économiques dans des liens informels apparaît comme un des facteurs de régulation des rapports sociaux. Si certains comportements ne sont pas admis et qu'ils sont répétés par certains acteurs, ces derniers risquent à la longue d'être exclus du réseau d'échanges économiques. Les occasions favorables pour ces acteurs diminuant d'autant, ils se trouvent forcés de réorienter leurs comportements. (Farrell, 2005).

À défaut de tels mécanismes de régulation, les acteurs ne travaillent pas ensemble, poursuivant isolément l'accroissement de leur seul intérêt. C'est cette situation que vivaient les boucanières avant 2003. Seuls quelques cas d'alliances informelles entre certaines boucanières nous empêchent de parler d'une situation de concurrence globale. L'historique des acteurs est souvent garant d'un capital de confiance ou non (Blumberg, 2001). Cependant, l'histoire des boucanières les a conduits tout droit dans une situation de méfiance mutuelle plutôt que de confiance. 
Selon Giullozo et Thépaut (2004), la CIE est un modèle organisationnel qui offre plus de flexibilité qu'une organisation corporative hiérarchique tout en réduisant les risques et les incertitudes d'une situation de marché. Elle permet en outre aux entreprises de se concentrer sur leurs forces et de confier certaines tâches aux partenaires ou encore à une structure issue de l'alliance. D'ailleurs, la

\section{Études de cas : historique}

Selon un rapport réalisé en 1985 sur l'industrie, le marché des boucanières était assez stable jusque dans les années 1970, mais, par la suite, l'arrivée d'un groupe d'acheteurs rend plus acharnée la compétition entre les boucanières (Co-fish consultants, 1985. Le rapport recommande donc aux exploitants de coordonner davantage leurs activités afin d'atténuer cette compétition nuisible à l'ensemble de l'industrie. Cette recommandation est formulée au moment où une association existe depuis 1984. C'est sur une base volontaire qu'on demandait aux boucanières de passer par la coopérative. Les ratés de l'association apparaissent clairement quelques années après sa création.

Le rapport formule donc une série de recommandations susceptibles d'améliorer la situation de compétition qui perdure malgré l'existence d'une association. Il est pertinent de rappeler que ce qui était alors proposé sera, à quelques nuances près, le modèle adopté en 2003 par l'association des boucanières. D'après le rapport, la solution la plus acceptable, est celle qui a le plus de chance de réussir, est que toutes les boucanières s'engagent à créer une agence de mise en marché en copropriété. L'entreprise devrait évaluer le marché des produits et recommander des quotas de production. Elle devrait acheter tout le poisson des producteurs et serait responsable de la vente du poisson. particularité de l'entente de coopération entre les boucanières a consisté en la création d'une entreprise. C'est le cas des boucanières qui se concentrent sur la production, alors que le groupe créé se charge de la commercialisation de la production ${ }^{6}$. En outre, la CIE permet aux entreprises de mieux se positionner sur le marché.

L'entreprise serait aussi chargée de créer un logo pour l'entreprise. À ce moment-là, sur les 19 boucanières membres de l'association, 17 avaient dit qu'elles accepteraient de parapher une telle entente. Le rapport recommande que s'il y avait formation d'une agence des ventes, il devait y avoir une marque commerciale unique ainsi qu'un objectif et des efforts communs. L'auteur du rapport croit que l'embauche d'un gérant permettrait d'assurer le suivi des décisions prises, afin de communiquer l'information et d'assurer la cohésion du groupe; il estime aussi qu'une approche collective peut prendre du temps à s'implanter. La confiance est cruciale pour assurer le respect d'une entente.

Une partie de l'étude est consacrée au rapport entre une telle association et le gouvernement. Le gouvernement provincial accorde des permis de production et il apporte un soutien financier à certaines boucanières. Étant donné la responsabilité du gouvernement provincial dans le développement de l'industrie, l'auteur de l'étude recommande que l'aide gouvernementale se concentre sur les membres de l'association, et ce, sur une base équitable. Une telle action inciterait l'industrie à mieux se coordonner et à travailler ensemble dans le cadre d'une telle agence de mise en marché.

Selon une autre étude réalisée en 2000 par une société conseil (Leblanc, 2000), 31 producteurs géraient 25 boucanières. 
L'industrie dans la région de Cap-Pelé embauchait 900 employés à temps partiel, représentant 438 emplois à temps plein. Durant les cinq années antérieures à l'étude, on estime la production annuelle à 2 millions de boîtes (à savoir 18000 tonnes américaines). Cependant, une étude de Pêches et Océans Canada (1987) montre qu'en 1986

\section{Le contrat et le mandat de l'association}

L'objet du présent contrat entre les boucanières vise la création d'une entreprise de mise en marché à laquelle sont liées 18 boucanières, sept demeurant indépendantes. Le contrat initial portait sur le prix de vente des produits et les quotas de production; il prévoyait de fortes amendes en cas de nonrespect des conditions de l'entente. Le prix de vente fixé est le même pour tout le monde. Les membres acceptent que tout le poisson soit vendu par l'association, autrement une lourde amende est prévue (150000\$). En limitant ainsi la portée du contrat, les promoteurs du projet voulaient s'assurer de faire signer le plus grand nombre de propriétaires possible. Ils ont donc écarté initialement une clause sur l'achat du poisson, car plusieurs boucanières entretenaient des rapports privilégiés avec des fournisseurs, qu'ils voulaient naturellement conserver.

La clause portant sur les quotas limitait la production à 50000 boîtes par boucanière. Elle était difficile à accepter pour certains, qui avaient une capacité de production supérieure, mais les membres ont compris que cet élément de l'entente pouvait être profitable à long terme en obtenant un meilleur prix de vente. C'est d'ailleurs ce qui s'est passé, puisque le prix de vente a presque doublé (répondant $\mathrm{n}^{\circ} 2$ ).

En outre, le gérant promettait que les quotas des boucanières seraient plus élevés la deuxième année. Il demandait que le contrat les 25 usines produisaient 1 million de boîtes de produits marins (soit 9000 tonnes), doublant leur production en quelques années et générant près de 7 millions \$ en 1986. Selon l'étude de Leblanc (2000), la valeur de la production s'élève à 40 millions $\$$. En 1986, la valeur de la production s'élevait à 15 millions \$, soit une hausse de $160 \%$.

fût signé pour une durée d'un an avant sa reconduction. Cette stratégie a convaincu plusieurs membres qui voyaient qu'elle ne constituait pas un grand risque, compte tenu de la situation de l'industrie du poisson fumé. $\mathrm{Au}$ lieu d'être reconduit pour deux ans, l'entente a été renouvelée jusqu'en 2010 et porte désormais autant sur la vente que sur l'achat. Chaque boucanière doit désormais passer par l'agence de mise en marché pour acheter le poisson, le bois, le sel, bref, les matières nécessaires pour la transformation du poisson. Cet étapisme a permis de convaincre les boucanières qui souhaitaient conserver leurs liens avec leurs fournisseurs. À cette fin, les perceptions devaient changer. Plutôt que de raisonner simplement en fonction de la qualité de leur produit, ou des liens de confiance qu'elles avaient personnellement développés avec leurs fournisseurs, elles ont peu à peu commencé à raisonner en fonction d'une logique collective, voyant que ce n'est plus tant la qualité de leur produit qui comptait, mais la qualité globale de production. En effet, même si chaque boucanière est identifiée sur les boîtes de produits, c'est d'abord le logo de l'agence de mise en marché qui ressort sur le produit (répondant $\mathrm{n}^{\mathrm{o}}$ 4). En outre, la compétition jouait toujours sur l'achat des poissons, ce qui faisait monter les prix de la ressource. Or, les boucanières commençaient à constater les avantages qu'il y avait à se regrouper pour lutter contre la concurrence (répondant $\mathrm{n}^{\mathrm{o}} 4$ ). Le respect du contrat 
apparaît comme une façon de dissiper la méfiance qui existe entre les boucaniers. Les boucanières plus vulnérables voyaient dans l'association un moyen leur permettant de résoudre leurs difficultés financières.

\section{L'importance d'un nouveau mode de communication comme facteur de confiance}

Pour établir un climat de confiance entre les signataires de l'entente, le gérant a dû instaurer un nouveau mode de communication. Il déploie tous les efforts pour amener les membres à régler aux réunions leurs différends, plutôt que d'attendre d'être dehors. Cette approche de la communication marque un changement par rapport aux relations qui prévalaient

\section{Une planification plus stable}

La passation d'un contrat associant les boucanières à une entreprise de mise en marché a permis de revoir l'organisation de la production. Chaque boucanière sait à l'avance quelle sera sa production annuelle du fait de l'existence de quotas. Par ailleurs, elle est assurée d'écouler sa production à un prix qui sera le même que celui de ses anciens concurrents. Ce système de planification réduit grandement l'incertitude pour les boucanières, au sujet notamment du prix de vente et de la possibilité de vendre. En outre, il permet de produire un poisson de meilleure qualité :

Aujourd'hui, grâce à l'agence de mise en marché, les boucanières contrôlent l'achat et la vente de produits marins. Plus d'une

\section{La formation d'un groupe}

La médiation par une entreprise de mise en assure une cohésion entre les entreprises, contribuant à éliminer la compétition qu'elles se livraient. Nous avons vu le rôle qu'exerce le contrat dans ce passage. Il joue un rôle incitatif central dans l'orientation des comportements des boucanières. Cependant, la contrainte tout comme l'intérêt, ne suffisent auparavant (répondant $n^{0} 7$ ). L'établissement d'un nouveau mode de communication joue un rôle dans l'instauration de relations de confiance entre les signataires de l'entente. Le fait de participer régulièrement à des réunions incite les membres du groupe à communiquer entre eux. Des liens de confiance se créent, contribuant à former un capital social qui peut s'avérer utile.

vingtaine de comités auxquels siègent plusieurs membres permettent de gérer un ensemble d'activités au sein de la nouvelle entreprise. Ces comités reposent sur l'engagement des boucanières membres de l'association, qui prennent ainsi en charge des tâches financières, le transport, la gestion de la production (quotas), l'achat de sel et de bois, les relations avec les membres, les licences, les questions juridiques et la qualité du poisson. La présence aux comités de membres provenant de plusieurs boucanières contribue à accroître leur confiance envers eux. Le gérant est informé des activités de chacun des comités, sur lequel il a droit de regard.

pas à expliquer le fonctionnement de cette coopération interentreprises. Des facteurs symboliques doivent favoriser le développement d'un sentiment d'appartenance. Le groupe des boucanières doit se former et exister en fonction d'éléments symboliques qui donnent corps au 
projet. Le groupe naît autour d'éléments symboliques qui l'expriment et l'identifient.

Pour donner naissance socialement au groupe, nous sommes en présence d'un échange symbolique éclairant entre le promoteur et les membres. D'une part, les membres du groupe tendent à faire reposer l'existence du groupe sur l'action du gérant, qui, en retour, fait reposer l'existence et le fonctionnement du groupe sur ses membres.

Cependant, pour les signataires de l'entente, c'est grâce aux qualités personnelles du gérant que le groupe existe et fonctionne. Plusieurs commentaires soulignent sa capacité à comprendre les personnes en les écoutant. Son sens de l'écoute lui a servi à saisir les enjeux entre les boucanières. Le fait que le gérant provient d'un milieu extérieur aux boucanières les a aidées à lui faire confiance. Le gérant insiste pour que les signataires de l'entente oublient querelles et conflits : s'il y a des membres qui résistent à un projet, alors il tente de le convaincre. On souligne sa fermeté et le fait qu'il sait où il va (répondant $\mathrm{n}^{\mathrm{o}} 4$ ) : le gérant insiste pour que la parole donnée et les ententes conclues soient respectées.

Bref, sur le plan symbolique, nous assistons à un don et à un contre-don de pouvoir : le groupe donne son pouvoir constitutif au gérant qui leur lui rend. Cependant, la formation $\mathrm{du}$ groupe demande un apprentissage. Le gérant insiste pour que le groupe «tourne la page » et « allume », c'està-dire que ses membres comprennent l'importance de changer leurs relations pour que l'association fonctionne.

\section{Le passage d'une logique individualiste à une logique collective}

L'existence du groupe repose sur le passage d'une logique individualiste à une logique collective de l'intérêt. Le passage d'une logique individualiste à une logique collective passe par un travail d'éducation grâce auquel selon le gérant chacun comprend que son sort est lié à celui de son partenaire. En outre, le gérant tente de favoriser un sentiment d'égalité entre les membres. Il invite les membres à voir ce que les boucanières ont en commun, plutôt que ce qui les distingue.

Mais la solidarité entre les membres demeure fragile. Un des répondants manifeste des résistances à l'égard de certaines pratiques du groupe. Il était d'accord pour signer une entente qui porte sur la vente. Le moment venu de faire porter l'objet de l'entente sur l'achat du poisson, il n'était plus d'accord. Il envisage de quitter l'association pour cette raison et reprendre ainsi la direction de son entreprise (répondant $\mathrm{n}^{\circ}$ 9)

Un autre répondant avait envisagé de joindre l'association, mais a refusé à la dernière minute. Il explique ses motifs parce qu'il n'avait pas confiance au gérant. Ce dernier n'aurait pas réussi à dissiper certains doutes concernant les garanties associées à la marge de crédit de l'association si les choses tournaient mal, alors que beaucoup de boucanières étaient dans une situation financière très difficile. Il doutait également de ce qui allait arriver avec ses marchés, qui étaient distincts de la majorité de ceux des autres boucanières. Nous voyons bien que la décision de faire partie du groupe demande que l'on accepte de remettre une partie de son indépendance à une entité qui sera créée pour prendre en charge certaines activités de vente d'abord, et d'achat ensuite. 


\section{Le rôle des symboles dans la formation du groupe}

L'existence du groupe se concrétisera dans l'emploi de symboles d'identification. C'est là un facteur déterminant dans la formation du groupe. L'adoption d'un logo unique pour représenter le groupe est une action qui exprime la reconnaissance des membres de l'association et leur engagement à lui donner une existence concrète. Par ailleurs, le gérant crée des pièces de vêtement (casquette, manteau ou T-shirt) aux couleurs de l'association et encourage les membres à les porter dans les rencontres et les activités de représentation.

\section{Éléments de réussite du groupe et menaces à l'horizon}

Le groupe des 18 boucanières est en meilleure position pour négocier l'achat des ressources requises pour la production et la vente des produits marins. De plus le groupe est intervenu à un palier politique pour bloquer l'allocation d'un nouveau permis. Une boucanière indépendante a voulu acheter la licence d'une boucanière qui avait cessé ses activités afin d'augmenter sa production. Plusieurs observateurs de l'industrie considèrent qu'une des causes de ses difficultés est la surproduction, ce qui a pour effet de faire diminuer les prix.

Une autre situation a permis de démontrer la force de la solidarité pour le groupe. Au début de la formation de l'association, un acheteur porte plainte au sujet de la qualité du produit. Cette stratégie fonctionnait auparavant alors que chaque boucanière était isolée. Cette fois cependant, plusieurs boucanières décident d'aller rencontrer les acheteurs sur place pour discuter de la qualité du produit. Cette stratégie des acheteurs s'est avérée infructueuse et n'a plus été employée par la suite.

\section{Conclusion}

Notre étude d'entreprises indépendantes en milieu rural permet de mettre en évidence les éléments constitutifs de création d'un mode de coopération interentreprises auparavant en forte concurrence. Le passage de rapports de méfiance à des rapports de confiance
Bref, les membres de l'association constatent les avantages qu'offre le groupe. Ils sont conscients du fait que les acteurs extérieurs ne peuvent plus profiter des dissensions et de la compétition qui prévalaient.

Aux yeux de ses membres, l'association constitue une réussite puisque, pour la première fois, on parvient à créer une association entre les entreprises qui, en outre, donne des résultats positifs. La constitution de l'association a permis à certains propriétaires de surmonter leurs difficultés économiques (répondant $\mathrm{n}^{\mathrm{o}} 4$ ). Mais, on pourrait penser que la création de l'association n'a fait que déplacer un vieux problème. La compétition et les relations conflictuelles se vivent maintenant entre les boucanières indépendantes et celles qui sont membres de l'association. D'ailleurs, un répondant d'une boucanière indépendante mentionne que, depuis la création de l'association, les indépendants se concertent davantage.

réciproques a été rendu possible par la conjonction de plusieurs facteurs :

- l'intervention d'une personne extérieure à l'industrie, 
- l'apprentissage d'un mode de communication fondé sur la transparence, le face-à-face et la franchise,

- un accès à l'information et le contrôle de la diffusion de l'information par le responsable en ce qui concerne la mise en œuvre de nouveaux projets,

- l'emploi de symboles désignant la CIE et cristallisant un nouveau sentiment d'appartenance et

- la passation d'un contrat formalisant les conditions de l'entente.

Le fait de voir les avantages qu'offre la coopération motive aussi les propriétaires à demeurer dans l'association. Parmi ces avantages, mentionnons

- les avantages économiques de la vente et de l'achat en groupe,

- le rapport de force que permet d'instaurer la solidarité entre les boucanières auprès des autres boucanières indépendantes et auprès du gouvernement ainsi que
- la stabilité de planification et la réduction de l'incertitude que permet la réduction des rapports de compétition entre les boucanières.

Soulignons que la réduction de l'incertitude constitue un élément central de la CIE : Plusieurs facteurs ont donc concouru à former un mode de CIE, mais ce qui demeure au fondement de cette forme de coopération, est l'échange symbolique du pouvoir entre le responsable de l'association d'entreprises et les membres de celle-ci. En accordant leur pouvoir au responsable de l'association que les propriétaires des boucanières créent un groupe social et exercent le pouvoir de le faire. C'est aussi dans la mesure où ce pouvoir est retourné aux membres que ceux-ci parviendront éventuellement à acquérir les capacités nécessaires pour se prendre en charge.

Ainsi on a pu voir que la CIE est une façon que les boucanières artisanales du territoire rural de Cap-Pelé a utilisé pour surmonter la compétition. Cependant ceci ne l'a pas éliminé mais l'a plutôt transposé à un autre niveau.

\section{Notes et références}

Pour respecter l'usage dans la région du sud-est du Nouveau-Brunswick, nous disons boucanière pour désigner une usine de harengs fumés et séchés.

2 En fait, les boucanières fument et salent plusieurs types de produits marins (gaspareau, morue, maquereau).

Entrevue réalisée avec les chercheurs.

4 Pour mener à bien notre étude, nous avons fait des entrevues semi-dirigées auprès de huit propriétaires de boucanières membres du regroupement et de un propriétaire de boucanière qui est demeuré indépendant. Nous avons également consulté des études portant sur l'industrie du hareng fumée et sur l'histoire de la région, ainsi que des documents fournis par les répondants.

5 Une boîte équivaut généralement à 18 livres.

6 Nous verrons plus loin que les responsabilités du groupe se sont accrues par la suite. 